\title{
Brecha digital y nuevas formas académicas en la escuela rural española durante el confinamiento
}

\author{
Carmen Álvarez-Álvarez \\ Universidad de Cantabria. España. \\ alvarezmc@unican.es
}

Francisco Javier García-Prieto

Universidad de Huelva. España.

fjavier.garcia@dedu.uhu.es

Recibido: 22/7/2020

Aceptado: 3/12/2020

Publicado: 5/7/2021

\section{Resumen}

La brecha digital dificulta la igualdad de oportunidades de la población. En este artículo nos preguntamos cómo afectó el confinamiento de la población y la docencia en línea a la escuela rural. Las conexiones son más deficientes y los recursos pueden ser escasos, pero la comunicación con las familias es fluida y los centros muestran una ratio menor. Hemos diseñado un cuestionario que ha sido respondido por 157 escuelas del medio rural durante el propio proceso de confinamiento. Los resultados indican una brecha digital para un 30\% del alumnado, notables esfuerzos por parte del profesorado para adaptarse a las necesidades educativas, una evolución semanal en la forma de afrontar la organización docente, propuestas didácticas afines y preocupación por la evaluación curricular. Las escuelas rurales participantes en el estudio han tratado de dar respuesta a las múltiples demandas que la nueva situación ha generado y de disminuir la brecha digital adaptándose a las necesidades de comunicación de sus estudiantes.

Palabras clave: escuela rural; TIC; docencia en línea; brecha digital; equidad; COVID-19

Resum. Bretxa digital $i$ noves formes acadèmiques a l'escola rural espanyola durant el confinament

La bretxa digital dificulta la igualtat d'oportunitats de la població. En aquest article ens preguntem com va afectar el confinament de la població i la docència en línia a l'escola rural. Les connexions són més deficients i els recursos poden ser escassos, però la comunicació amb les famílies és fluida i les escoles mostren una ràtio més baixa. Hem dissenyat un qüestionari que ha estat respost per 157 escoles del medi rural durant el propi procés de confinament. Els resultats indiquen una bretxa digital per a un 30\% de l'alumnat, notables esforços per part del professorat per adaptar-se a les necessitats dels infants, una evolució setmanal en la manera d'afrontar l'organització docent, propostes didàctiques afins i preocupació per l'avaluació curricular. Les escoles rurals participants en l'estudi han tractat de donar resposta a les múltiples demandes que la nova situació ha generat i disminuir la bretxa digital adaptant-se a les necessitats de comunicació amb cadascun dels seus estudiants.

Paraules clau: escola rural; TIC; docència en línia; bretxa digital; equitat; COVID-19 
Abstract. The Digital Gap and New Academic Forms in Rural Schools of Spain During Lockdown

The digital divide constitutes a barrier to achieving equal opportunities for the population. In this article, we examine how the lockdown and online teaching affected rural schools. Although connectivity is poor and resources may be scarce in these schools, communication with families is fluid and the teacher-student ratio is lower. We designed a questionnaire that was administered to 157 rural schools during the lockdown process. The results show a digital gap for $30 \%$ of the student body, notable efforts by teachers to adapt to the needs of students, weekly changes in the way teaching is organized, related didactic proposals, and concern for curricular assessment. The rural schools participating in the study have tried to respond to the multiple demands arising from this new situation and narrow the digital gap by adapting to the communication needs of their students.

Keywords: rural school; ICT; online teaching; digital gap; equity; COVID-19

\author{
Sumario \\ 1. Introducción 4. Conclusiones \\ 2. Método Referencias bibliográficas
}

3. Resultados

\title{
1. Introducción
}

Estudios realizados en diversos países han puesto de manifiesto la relevancia de que las autoridades equipen a los centros educativos rurales de todo el mundo con conexión a Internet en el máximo de áreas posible y que las nuevas tecnologías sean empleadas regularmente con los estudiantes de poblaciones pequeñas para evitar que haya una brecha digital territorial que niegue oportunidades educativas al alumnado rural (Arancibia y Carrasco, 2006; Halili y Sulaiman, 2019; Molina y Mesa, 2019; Moral, Villalustre y Neira, 2014; Morales, 2018; Mwapwele, Marais, Dlamini y Van Biljon, 2019; Raso, Hinojo y Sola 2015; Wang, Tigelaar y Admiraal, 2019).

La brecha digital puede ser a tres niveles: acceso a las TIC, utilización de las TIC y conocimientos en materia de TIC (Hu, Gong, Lai y Leung, 2018). En cuanto al acceso a las TIC, diversos estudios han puesto de manifiesto que las escuelas rurales tienen más dificultades para contar con recursos tecnológicos que las urbanas, sobre todo buenas conexiones, por lo que existe una brecha digital mayor entre los estudiantes (Arancibia y Carrasco, 2006; Bai et al., 2016; Kim, Kil y Shin, 2014; Molina y Mesa, 2019). En ocasiones esto se ha paliado a través de conexiones públicas comunitarias en los pueblos. Un estudio realizado con una muestra de estudiantes de Primaria en 173 escuelas coreanas demostró que la alfabetización en materia de TIC de los centros educativos situados en las principales ciudades fue superior al nivel de los pueblos pequeños, por lo que se recomendó que las autoridades facilitaran que 
en el medio rural se dispusiera de un ordenador por escolar (Kim et al., 2014). Esta medida, implantada en diferentes países, fue evaluada en Brasil y mostró un cambio actitudinal en los profesores, los estudiantes y los familiares: se produjo una progresiva formación e integración curricular por parte de los docentes, una mayor autonomía del alumnado y un avance alfabetizador en el empleo de tecnología por parte de los padres y las madres (Pescador y Valentini, 2019).

En cuanto a la utilización de las TIC en la educación rural, se ha defendido principalmente que la tecnología fuera integrada curricularmente para promover y fortalecer los procesos de enseñanza-aprendizaje (Arancibia y Carrasco, 2006; Bai et al., 2016; Molina y Mesa, 2019; Moral et al., 2014). También se ha abogado para que la utilización de la tecnología por parte de los centros sirva para estar en contacto con las familias e introducir a las nuevas generaciones en su manejo para generar hábitos de uso (más allá de emplearla en situaciones excepcionales), promoviendo la aceptación de los estudiantes rurales del manejo de las TIC y su competencia digital (Halili y Sulaiman, 2019). Asimismo, también se ha defendido que los centros elaboren un plan estratégico para la incorporación de las TIC donde se presenten sus posibilidades de conectividad, su infraestructura tecnológica y sus necesidades de capacitación y transferencia tecnológica (Molina y Mesa, 2019).

El uso de las TIC en las escuelas rurales está estrechamente ligado con la dotación tecnológica del centro, pero también con la actitud docente ante la tecnología y su formación específica al respecto. Un estudio realizado en China demostró que lo que influía de manera más significativa en el uso de recursos educativos digitales en la escuela eran las actitudes, los conocimientos y las habilidades de los docentes (Wang et al., 2019). En las áreas rurales es deseable contar con profesorado especialmente formado y sensible respecto a la necesidad de dar el máximo de oportunidades al alumnado para contribuir a erradicar su posible situación inicial de desventaja (Andreea-Diana, 2014; Arancibia y Carrasco, 2006; Lozoya, Quintana, Armenta y Pérez, 2018; Molina y Mesa, 2019; Moral et al., 2014; Morales, 2018). Un estudio realizado en España con profesorado de centros rurales y urbanos concluyó que la formación inicial de los enseñantes de niveles no universitarios en medios y tecnologías es, en general, escasa, y aquellos que la han recibido declaran que ha tenido un marcado carácter teórico y su utilidad es nula o escasa (Ortega et al., 2005). En otro estudio reciente realizado con directores españoles, estos demandan una mejor dotación en tecnologías de la información y la comunicación (Álvarez-Álvarez et al., 2020).

La literatura ha demostrado que el uso de las TIC en el aula y en el hogar influye de manera diferente en el aprendizaje de los estudiantes. Una investigación basada en los datos de TIMSS 2011 y PIRLS 2011 identificó que el uso de las TIC tanto en la escuela como en el hogar influía positivamente en el rendimiento de los estudiantes de cuarto grado en lectura, matemáticas y ciencias (Skryabin, Zhang, Liu y Zhang, 2015). Sin embargo, otra investigación más reciente (Hu et al., 2018), realizada en base a datos de PISA, reveló 
que la disponibilidad de TIC de los estudiantes en la escuela se asocia positivamente a su éxito académico, mientras que la disponibilidad en el hogar se asocia negativamente a él. Esto sucede porque el uso de la tecnología en el hogar está más dirigido al entretenimiento que a la formación (Petko, Cantieni y Prasse, 2017).

Asimismo, en el ámbito rural existe una brecha digital en los hogares ligada al plano socioeconómico que afecta tanto a la conectividad a internet como al equipamiento tecnológico y a la formación, como sucede con las escuelas. El estudio realizado en Colombia en diez centros educativos de la zona de Tunja muestra que sus alumnos provienen de familias de bajos recursos, lo que hace poco probable la prevalencia de dispositivos tecnológicos como computadores, tabletas y acceso a internet en sus hogares. Igualmente, estos investigadores también concluyen que, aunque se ha invertido en infraestructura, el acceso a internet de forma permanente y de calidad en las áreas rurales no se ha logrado materializar y que los fallos eléctricos son frecuentes (Molina y Mesa, 2019). Otro estudio, de tipo comparativo, llevado a cabo en Canadá, Nueva Zelanda y Australia realizado a partir de los datos de PISA, demostró que los entornos de aprendizaje de las escuelas son menos positivos en las comunidades rurales que en las ciudades, y esto se debe en buena medida al estatus socioeconómico, que es más alto en las grandes poblaciones y, por tanto, es más probable que las familias que viven en ellas provean de libros, ordenadores, material educativo y medios tecnológicos a sus hijos, por lo que las escuelas desempeñan un papel crucial para salvar la brecha socioeconómica y digital existente entre el campo y la ciudad y promover la igualdad de oportunidades y la equidad (Sullivan, McConney y Perry, 2018).

En España, aunque la accesibilidad a internet ha mejorado en los últimos años, sobre todo con la generalización del uso de teléfonos inteligentes, aún hay ciudadanos excluidos de la sociedad de la información (Gallardo-Echenique, 2019), y este dato es más alarmante cuanto menores son los ingresos de las familias. Gran número de hogares españoles con ingresos mensuales inferiores a 900 euros disponen de teléfono móvil $(95,5 \%)$, pero no cuentan con ordenador (incluidos portátiles, tabletas, etc.) en un porcentaje medio del $58 \%$, aunque hay grandes diferencias territoriales, por ejemplo, entre la cifra más baja de Ceuta, del 39,6\%, y la más alta de Navarra, del 80\%. Los hogares españoles con ingresos mensuales inferiores a 900 euros disponen de acceso a internet en un $77,9 \%$ de los casos (el porcentaje más bajo corresponde a Ceuta —39,6\%—, y el más alto, a Madrid — 84,5\%—) y el porcentaje aumenta a medida que los ingresos familiares también aumentan (Instituto Nacional de Estadística, 2019). Otro estudio realizado en Granada (España) ha revelado que casi todos los docentes (un 94,4\%) poseen un ordenador personal y que casi un $90 \%$ (un 88,2\%) además están conectados a Internet en su domicilio, y más de un 65\% (un 66,7\%) los que tienen, asimismo, un ordenador portátil, teniendo cierta competencia técnica, aunque en la docencia en muchos casos no vayan más allá de elaborar apuntes o crear archivos de PowerPoint 
(Raso et al., 2015). En lo relativo a la velocidad de Internet en las áreas rurales, no suele ser alta y hay algunas que ni siquiera tienen acceso a la red.

Como consecuencia de la pandemia de COVID-19 muchos países han cerrado las instituciones educativas, al ser espacios donde se producen permanentemente interacciones sociales, lo que podría contribuir a elevar significativamente los contagios (Rus, Sandu y Tasen e, 2020). En España esta medida adoptada por el Gobierno se inició el 16 de marzo de 2020 y afectó a todos los estudiantes hasta el final de curso. Durante el periodo de confinamiento obligatorio decretado en el país, muchas escuelas pusieron de manifiesto que no todos los alumnos podían seguir una docencia en línea en casa por diferentes razones: el centro no disponía de una plataforma de teleformación en uso, falta de conexión a internet en casa, carencia de medios tecnológicos, ausencia de formación tecnológica familiar, etc. (Marquès, 2020). En la escuela rural podríamos estar ante una triple exclusión: brecha digital (mayores dificultades de acceso a las TIC), brecha territorial (utilización de las TIC y conocimientos en materia de TIC menos extendidos que en el mundo urbano) y brecha socioeconómica (mayor riqueza de medios en el mundo urbano).

Esta situación lleva a preguntarnos qué posibilidades y qué dificultades han tenido las escuelas del medio rural ante un periodo obligado de docencia en línea. ¿Cómo lo han vivido y cómo han actuado? ¿Los centros se han organizado efectivamente? ¿Se han buscado soluciones adaptadas a cada caso? ¿Qué elementos han sido de utilidad y cuáles han supuesto una dificultad? ¿Qué cabe mejorar en el sistema educativo de cara a afrontar crisis como esta que estamos viviendo para estar mejor preparados?

\section{Método}

Nuestra hipótesis de partida es que existe una brecha digital en el ámbito rural a varios niveles que puede actuar como un elemento entorpecedor en este proceso de docencia en línea, pero pensamos que hay otros factores que pueden ayudar a suplir estas carencias, ya que en España la escuela rural tiene una ratio baja y la comunicación con las familias es fluida (Álvarez-Álvarez et al., 2020), con lo que se pueden compensar algunas deficiencias y el centro docente puede desempeñar un papel relevante en la igualdad de oportunidades.

Nuestro objetivo es conocer cómo los centros rurales han organizado los medios a su disposición para afrontar el periodo de confinamiento de los estudiantes en sus casas en España. Son objetivos específicos: (1) indagar sobre la brecha digital en los centros y en las familias y conocer las posibilidades de acceso telemático de los estudiantes, y (2) conocer las nuevas formas académicas a nivel de organización docente, propuestas didácticas implementadas y evaluación curricular.

Para ello se ha planteado un estudio durante el periodo de confinamiento con un fin exploratorio, descriptivo e interpretativo. Hemos diseñado un cuestionario en línea compuesto por preguntas de diferentes tipologías: (1) cuestiones de perfil sociodemográfico y educativo; (2) preguntas dicotómicas de 
elección única establecidas, que han sido útiles en la medida en que no se establece la tendencia a la media y son las mayoritarias; (3) preguntas acumulativas de opción múltiple —no son excluyentes entre sí-; (4) preguntas de elección única politómicas; (5) preguntas de escalas numéricas y nominales; (6) preguntas abiertas, y (7) preguntas cerradas. Se ha invitado por correo electrónico a colaborar a todos los centros rurales españoles. La recogida de la información ha sido digital. Para su validez de contenido y constructo se llevó a cabo una prueba de juicio de expertos con 10 docentes rurales.

La muestra estudiada la componen 306 docentes (157 escuelas participantes) del medio rural en España, el 73,2\% de los cuales corresponde a mujeres $(n=224)$, y el $26,8 \%$, a hombres $(n=82)$. La media de edad de los participantes se sitúa en los 41,28 años. Respecto a la situación administrativa, el 62,5\% es definitivo; el $14,5 \%$, provisional, y el $23 \%$, interino. Los datos han sido extraídos entre el 25 de marzo y el 25 de abril de 2020 . Se han obtenido respuestas de todas las comunidades autónomas a excepción de Madrid (tabla 1).

A través del software SPSS v. 21 (exportado desde Excel) se han realizado análisis descriptivos básicos. En los datos se han tenido en cuenta los distintos protocolos de colaboración anónima, imparcialidad y protección de datos.

Tabla 1. Centros educativos que han colaborado por comunidades

\begin{tabular}{lcc}
\hline Comunidad autónoma & Participantes (\%) & Escuelas \\
\hline Andalucía & 16,1 & 25 \\
\hline Aragón & 5,8 & 9 \\
\hline Baleares & 1,9 & 3 \\
\hline Canarias & 11,6 & 18 \\
\hline Cantabria & 6,5 & 10 \\
\hline Castilla y León & 1,9 & 3 \\
\hline Castilla-La Mancha & 6,5 & 10 \\
\hline Cataluña & 1,3 & 2 \\
\hline Comunidad Foral de Navarra & 2,6 & 4 \\
\hline Comunidad Valenciana & 21,3 & 33 \\
\hline Extremadura & 4,5 & 7 \\
\hline Galicia & 3,2 & 5 \\
\hline La Rioja & 1,3 & 2 \\
\hline País Vasco & 7,7 & 12 \\
\hline Principado de Asturias & 3,9 & 6 \\
\hline Región de Murcia & 3,9 & 6 \\
\hline Total & & 157 \\
\hline
\end{tabular}

Fuente: elaboración propia. 


\section{Resultados}

La enseñanza telemática en la educación rural ha significado un replanteamiento de la docencia. En coherencia con nuestros objetivos específicos, nos centraremos en dos aspectos: (1) indagar sobre la brecha digital para conocer las posibilidades de acceso telemático de los estudiantes y (2) conocer las nuevas formas académicas.

\subsection{Brecha digital}

La nueva situación ha generado preocupación en la comunidad docente sobre la posible acentuación de las desigualdades socioeducativas. Para analizar este aspecto nos detendremos en el acceso telemático y en los recursos disponibles en el hogar, así como en el seguimiento de los estudiantes.

\section{a) Acceso telemático y recursos en el hogar}

Para el 49\% del profesorado de la escuela rural el acceso a Internet en su pueblo es limitado y señala como la principal causa la calidad de las conexiones (un $52,96 \%$ piensa que son malas o muy malas).

Los centros participantes en nuestro estudio tienen una ratio media de 12,66 escolares por aula, de los cuales 3,79 (un 29,9\%) no tienen acceso a internet o posibilidades de conexión telemática, lo que imposibilita la igualdad de oportunidades durante el confinamiento.

El $91,5 \%$ de profesores mantiene contactos semanales con las familias a través de grupos de aplicaciones de teléfono inteligente o correo electrónico. De estos, el $46 \%$ se produce a través de videollamadas.

Respecto a la accesibilidad y al equipamiento, el alumnado ha contado con los siguientes dispositivos (no excluyentes entre sí):

- Ordenador familiar: un $85 \%$.

- Teléfono inteligente: un 70,7\%.

- Ordenador personal: un $17 \%$.

Un 30\% no posee ordenadores en el domicilio, ello coincide con el dato de que el $29,9 \%$ de los estudiantes no tiene acceso a internet. Desde el punto de vista del profesorado, un $41,1 \%$ de las familias no tiene conocimiento sobre el uso de las TIC.

Los participantes han señalado que el alumnado cuyas familias han trabajado fuera del hogar durante el confinamiento (un 81,5\%) ha visto reducidas las posibilidades de aprendizaje, ya que los escolares dependen del teléfono de algún familiar para hacer las tareas académicas o conectarse a videoconferencias. El 54,2\% de los padres y madres han mostrado preocupación por avanzar contenidos. 


\section{b) Seguimiento de los estudiantes}

La enseñanza telemática ha generado incertidumbre e inseguridad entre el profesorado de las escuelas rurales, y las limitaciones tecnológicas en los hogares ha supuesto una mayor intensificación laboral para el 85,6\%. Asimismo, el $38,5 \%$ de los participantes consideran que se ha cargado excesivamente de tareas al alumnado en las diferentes asignaturas. Los maestros coinciden en afirmar que la comunicación con los escolares es buena (67,9\%), pero nuevamente se observa que hay un porcentaje (un 32,1\%) de estudiantes que está completamente fuera de los procedimientos generales llevados a cabo, bien sea por falta de acceso, de dispositivos o de interés familiar.

Con el alumnado que no tiene acceso telemático para avanzar académicamente, el profesorado ha llevado a cabo diferentes procedimientos:

- El 28,2\% hace un seguimiento telefónico semanal de cada estudiante.

- El 14,1\% recomienda a la familia que siga el libro de texto y que realice actividades que el docente va dosificando por diferentes vías.

- El 9,4\% da libertad a las familias para que se organicen como mejor consideren.

- El 4\% envía el material del trabajo por correo postal.

- El 8\% se ha resignado y ha admitido que en los pueblos no se puede hacer nada más allá de pequeñas acciones y que, por tanto, una parte importante de la población escolar quedaría al margen.

- En casos puntuales se han remitido las tareas escolares por medio de ayuntamientos $(6,3 \%)$, servicios sociales, protección civil y policía tutor.

Los participantes destacan que estas instituciones y figuras han sido claves, puesto que han proporcionado rúteres, material informático e incluso fotocopias que hacen llegar a esos domicilios (las correcciones se realizan por teléfono). Asimismo, por las respuestas abiertas, sabemos que vecinos que disponen de wifi han cedido sus claves a escolares y algunas escuelas rurales han prestado sus miniordenadores y tabletas a los estudiantes.

El esfuerzo de los docentes ha sido relevante, pero hay situaciones que exceden de su marco de acción: la brecha digital está acentuando una brecha académica. El 98\% del profesorado considera que esta crisis está afectando negativamente en mayor medida al alumnado más vulnerable.

\subsection{Nuevas formas académicas}

Las dinámicas de enseñanza desarrolladas se articulan en torno a tres ejes: a) organización docente, $b$ ) propuestas didácticas y $c$ ) evaluación curricular.

\section{a) Organización docente}

Las propuestas docentes han evolucionado durante el confinamiento, de modo que se han visto influenciadas por las normativas desarrolladas por las instituciones, las decisiones tomadas por los claustros, así como por la formación del 
profesorado. Los centros que han colaborado en este estudio han señalado lo siguiente:

- Un 40,8\% indica que está llevando a cabo una misma propuesta docente.

- Un $28,3 \%$ señala que tiene una propuesta consensuada a nivel de centro, pero varía el compromiso de cada enseñante.

- Un 24,3\% revela que no hay una propuesta conjunta a nivel de centro y que se está desarrollando la docencia telemática bajo una organización individual.

- Un 6,6\% revela otras formas organizativas en las que suele primar el trabajo en subgrupos docentes, adaptando acuerdos con el ciclo o el grupo, a fin de evitar el aislamiento.

Las respuestas recibidas (entre el 25 de marzo y el 25 de abril de 2020) permiten ver las siguientes tendencias temporales en la organización docente de los centros.

Tabla 2. Evolución de la organización docente por semanas

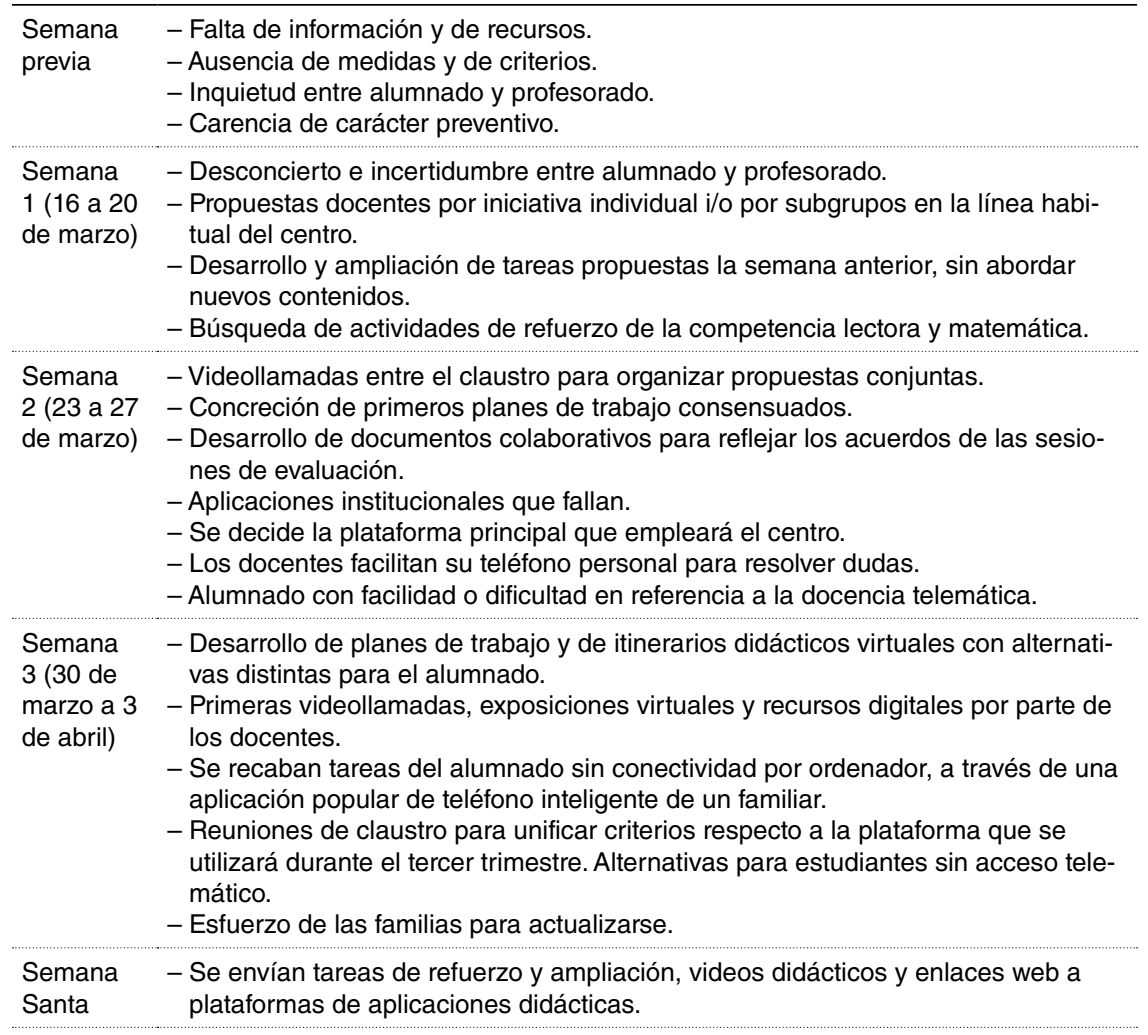




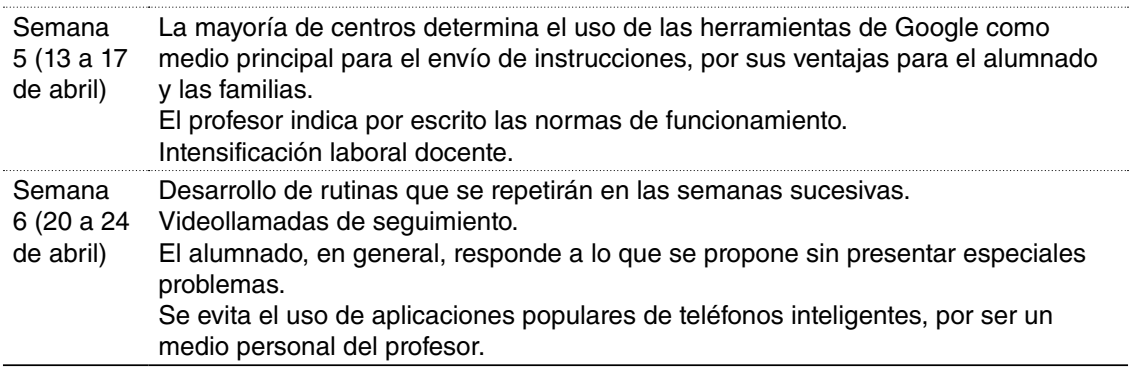

Fuente: elaboración propia.

El 79\% de los docentes realiza un seguimiento del alumnado: cuando el estudiante tiene acceso telemático se emplea mayoritariamente el correo electrónico (un 38,5\%), y cuando no tiene, se opta principalmente por el teléfono. Una amplia mayoría de los docentes (un 84,9\%) piensa que los escolares están aprovechando las actividades. De hecho, el $80,3 \%$ afirma que las tareas propuestas se realizan en el tiempo y la forma deseados.

Las plataformas, aplicaciones y herramientas telemáticas han sido diversas. En preguntas de elección múltiple que no son excluyentes entre sí, los participantes indicaron los siguientes datos:

- El 26,1\% usa plataformas en línea. Desconocemos por qué no se da un uso más mayoritario de este tipo de plataformas.

- El 60,1\% utiliza blogs y webs específicas.

- El 87,5\% emplea el correo electrónico como medio fundamental para el desarrollo de su enseñanza telemática.

- El 40,5\% se sirve de aplicaciones para hacer videoconferencias.

- El 36,6\% aprovecha la herramienta de Google.

- El 56,2\% utiliza aplicaciones de Google o de otras instituciones.

- El 69,2\% emplea WhatsApp y el 20,2\% opta por Telegram (ambas son aplicaciones populares en los teléfonos inteligentes de toda la población en 2020). Alrededor de un $10 \%$ de la muestra no utiliza ninguna de las dos aplicaciones.

\section{b) Propuestas didácticas}

Por otra parte, las propuestas didácticas en este periodo han seguido ciertos patrones. Por orden de frecuencia, se han identificado los siguientes:

1. Se envían tareas junto con un vídeo explicativo a través de correo, se resuelven dudas, se corrige la tarea y se realiza una retroalimentación posterior.

2. Se sube la tarea a una plataforma o herramienta con los audios y/o los vídeos correspondientes, y el alumnado debe depositar la actividad elaborada notificando fechas y subidas por correo y/o aplicaciones para teléfonos inteligentes. 
3. Envío de una programación semanal con distintas tareas a realizar durante cada jornada. Al final de semana los estudiantes entregan su dosier con las actividades.

4. Videoconferencia y planteamiento de actividad, seguida de una reflexión compartida como evaluación.

5. Se suben las tareas al blog, se manda el enlace correspondiente por aplicaciones populares de teléfonos inteligentes o por correo, se corrigen y se envían las correcciones por escrito o por audio.

El $81,7 \%$ de los docentes rurales sigue una secuencia didáctica que se estructura en dos tiempos: (1) requerir una tarea específica y (2) subir las correcciones en alguna aplicación indicada. Respecto al tipo de actividades, se especifican en la tabla 3 .

Tabla 3. Tipo de actividades propuestas

\begin{tabular}{lc}
\hline Actividades propuestas (no excluyentes) & Porcentaje \\
\hline Actividades elaboradas por el profesorado & $82,2 \%$ \\
\hline Visualización de vídeos didácticos y resolución de actividades & $72,4 \%$ \\
\hline Actividades en webs y/o blogs & $64,5 \%$ \\
\hline Fichas & $61,8 \%$ \\
\hline Lecturas obligatoria o voluntarias & $60,5 \%$ \\
\hline Ejercicios de libros de texto & $52,6 \%$ \\
\hline Actividades en equipo con otros compañeros & $30,3 \%$ \\
\hline
\end{tabular}

Fuente: elaboración propia.

Otras acciones puntuales han sido: Kahoot!, juegos en línea, Snappet, pasatiempos, fichas interactivas, exposiciones orales de temas trabajados, blogs colaborativos, experimentos, murales o maquetas. Añadido a ello se ha estimulado a los escolares a ver programas educativos en el hogar, como, por ejemplo, parte de la programación de Clan TV.

Estas actividades se trabajan con un contenido específico al que accede el alumnado y que es presentado bajo diferentes formatos: textos (un 86,9\%), vídeos (un 93,5\%), audios (un 66,7\%) e infografías (un 33,3\%). Estas tareas son trabajadas por los escolares individualmente (un 72,5\%), y una amplia mayoría indica que se establecen secuencias interactivas (un 73,8\%).

Todo este tipo de enseñanza genera incertidumbre entre los profesores respecto a su impacto (un 74,5\%). Ellos manifiestan un esfuerzo por abarcar a todo el alumnado (un 93,4\%). Los docentes consideran que no están enviando demasiadas tareas (un 86,2\%), lo cual contrasta con la percepción que tienen las familias (el $49 \%$ de los maestros identifica angustia en los hogares por el frecuente envío de tareas).

Hay una amplia coincidencia en los participantes en este estudio: este tipo de enseñanza telemática propicia menos aprendizajes que las clases presencia- 
les (un 90,8\%), a lo cual se añade la mala calidad de algunas conexiones a internet. El 62,7\% de los maestros indica que el contexto rural no es el medio más adecuado para aprender vía telemática. El 61,4\% del profesorado considera que lleva a cabo propuestas metodológicas telemáticas diferentes a las que se conocen en las escuelas urbanas.

\section{c) Evaluación curricular}

La evaluación de la adquisición de los contenidos influye notablemente en la enseñanza telemática. Al profesorado le preocupa bastante (3,21 en una escala tipo Likert del 0 - nada - al 5 - totalmente-) que el alumnado no adquiera los conocimientos previstos para el curso. De hecho, el 30,5\% de los docentes ha marcado un 5 en la escala. El 49,6\% de los enseñantes piensa que los escolares no van a aprender contenidos fundamentales este curso.

Para evaluar la adquisición de contenidos se siguen diversos procedimientos. El 86,3\% del profesorado realiza un seguimiento de las tareas y devuelve las correcciones. Las mismas se envían a la familia diariamente (un 35,8\%), semanalmente (un $41,1 \%$ ), no se envían (un 7,9\%), son autocorregibles o se pide su corrección a la familia, etc. (un 15,2\%).

La mayoría de estas correcciones son remitidas por diferentes vías, en función del manejo tecnológico de los estudiantes y de las familias (tabla 4).

Tabla 4. Herramientas utilizadas para el envío de correcciones y/o para evaluaciones

\begin{tabular}{lc}
\hline Herramienta para la corrección y/o la evaluación & Porcentaje \\
\hline Correo electrónico & $66,4 \%$ \\
\hline Aplicaciones populares para teléfonos inteligentes & $47,9 \%$ \\
\hline Aplicación o herramienta institucional & $24,7 \%$ \\
\hline Aplicación o herramienta libre & $13 \%$ \\
\hline
\end{tabular}

Fuente: elaboración propia.

Aunque no es generalizable, destaca que el $13,7 \%$ ha realizado exámenes tipo test en línea a través de alguna aplicación o herramienta.

\section{Conclusiones}

Los resultados permiten establecer algunas conclusiones provisionales, a la espera de más estudios que refrenden o contradigan los datos obtenidos.

En primer lugar, se verifica la existencia de una brecha digital y a diferentes niveles en el mundo rural (alrededor del 30\%) que afecta en negativo al aprendizaje en línea por parte de las escuelas rurales. Sigue siendo, por tanto, necesario dotar tecnológicamente a los centros, mejorar el acceso a internet en los pueblos para superar su aislamiento digital y la desigualdad educativa. Además, los docentes expresan que casi la mitad de familias no tienen conocimientos mínimos sobre aplicaciones y herramientas TIC. Cabe destacar que 
el correo electrónico y las aplicaciones populares en los teléfonos inteligentes han contribuido a minimizar la brecha digital y la exclusión (Gallardo-Echenique, 2019), lo que ha facilitado el contacto directo entre el profesorado y la familia para tener mayor alcance en el seguimiento del alumnado. Su uso está más generalizado que contratar internet en casa o tener ordenadores personales. Ello confirma nuestra hipótesis de partida y coincide con resultados de estudios previos en el panorama nacional e internacional (Bai et al., 2016; Carreño, 2015; Halili y Sulaiman, 2019; Hu et al., 2018; Kim et al., 2014; Lozoya et al., 2018; Molina y Mesa, 2019; Moral et al., 2014; Morales, 2018; Mwapwele et al., 2019; Raso et al., 2015; Wang et al., 2019).

En segundo lugar, ha sido posible conocer las formas académicas en cuanto a organización docente, propuestas didácticas y evaluación curricular. Tradicionalmente, los maestros rurales se han caracterizado por su capacidad de adaptarse a situaciones divergentes (Álvarez-Álvarez et al., 2020). Para el alumnado que no ha contado con acceso telemático, se han explorado diversas acciones: seguimiento telefónico con indicaciones semanales, tareas del libro de texto, correcciones diarias o semanales, etc. Por otra parte, algunas instituciones han facilitado material curricular e informático a escolares en riesgo de abandono escolar. A nivel de centro, más de dos tercios de los claustros han estado coordinados, aunados bajo una misma propuesta docente de enseñanza telemática. La mayoría de los docentes rurales considera que este periodo se está aprovechando a nivel curricular, pero también opinan que se deben mejorar el acceso a las TIC y proporcionar los medios necesarios a las familias rurales, ya que la escuela debe fomentar la igualdad de oportunidades sin dejar fuera al alumnado más desfavorecido. En relación con los contenidos, los maestros muestran preocupación por su desarrollo. La mayoría han optado por correcciones de actividades, siendo minoritarios los exámenes como medio para valorar los conocimientos del alumnado. El profesorado rural ha sentido mayor carga laboral, ansiedad frente al uso de nuevas aplicaciones, inseguridad en relación con las propuestas y los itinerarios didácticos planteados, etc., a la par que conocía, analizaba y experimentaba nuevas posibilidades de aprendizaje mediante herramientas digitales. Esto confirma nuestra hipótesis de que la baja ratio y la comunicación fluida con las familias ha contribuido a disminuir la brecha digital y académica que podía haber supuesto el periodo de confinamiento. Es necesaria mayor investigación, por ser un fenómeno nuevo que no dispone de literatura científica.

La distribución asimétrica de los participantes, los sesgos de disponibilidad o la baja representatividad, así como los aspectos propios del instrumento y los distintos estadios por los que han pasado los participantes limitan este estudio, que ofrece una radiografía de un tiempo y de unos centros determinados. Como línea de continuidad se está planteando un análisis mediante un protocolo de entrevista telefónica seleccionado a través de un muestreo por conveniencia a familias y estudiantes. 


\section{Referencias bibliográficas}

Álvarez-Álvarez, C.; García, J. y Pozuelos, F.J. (2020). Posibilidades, limitaciones y demandas de los centros educativos del medio rural en el norte y sur de España contemplados desde la dirección escolar. Perfiles Educativos, 29, 13-16.

AndreEa-Diana, S. (2014). The Importance of Involving Pupils from the Rural Area in Using ICT Skills and Tools: A Milestone. Procedia: Social and Behavioral Sciences, 128, 36-43.

$<$ https://doi.org/10.1016/j.sbspro.2014.03.114>

Arancibia, M. y Carrasco, Y. (2006). Incorporation of computers in rural schools: A descriptive study of four cases in the south of Chile. Estudios Pedagógicos, 32(2), 7-26.

Bai, Y.; Mo, D.; Zhang, L.; Boswell, M. y Rozelle, S. (2016). The impact of integrating ICT with teaching: Evidence from a randomized controlled trial in rural schools in China. Computers and Education, 96, 1-14. <https://doi.org/10.1016/j.compedu.2016.02.005>

Carreño, F.F. (2015). Experiencias de los docentes en la implementación de las TIC en escuelas rurales multigrado / Teachers experiencies in ICT implementation at multigrade rural school. Revista de Educacion Mediatica y TIC, 21.

Gallardo-Echenique, E.E. (2019). Brechas y asimetrías que emergen en la era digital: ¿Nuevas formas de exclusión? Revista Electrónica de Investigación Educativa, 21(3), 1-3. Recuperado de <http://redie.uabc. mx/redie/article/view/2909>.

Halili, S.H. y Sulaiman, H. (2019). Factors influencing the rural students' acceptance of using ICT for educational purposes. Kasetsart Journal of Social Sciences, 40(3), 574-579.

<https://doi.org/10.1016/J.KJSS.2017.12.022>

Hu, X.; Gong, Y.; LaI, C. y Leung, F.K.S. (2018). The relationship between ICT and student literacy in mathematics, reading, and science across 44 countries: A multilevel analysis. Computers and Education, 125 (junio), 1-13. <https://doi.org/10.1016/j.compedu.2018.05.021>

Instituto Nacional de Estadística (2019). Encuesta sobre Equipamiento y Uso de Tecnologias de Información y Comunicación en los hogares 2019. Recuperado de <https://www.ine.es/up/OhOvYvd5i4>.

KIm, H.S.; KIL, H.J. y SHIN, A. (2014). An analysis of variables affecting the ICT literacy level of Korean elementary school students. Computers and Education, 77, 29-38. <https://doi.org/10.1016/j.compedu.2014.04.009>

Lozoya, S.V.M.; Quintana, J.T.; Armenta, J.A. y Pérez, M.V. (2018). Contextual attribute variables in the use of ICT in primary level students from Southern Sonora, Mexico. Estudios sobre Educacion, 35, 499-515. <https://doi.org/10.15581/004.35.499-515 >

MARQuÈs, P. (2020). Estudio para conocer las actuaciones realizadas por los centros para proporcionar formación on-line a los estudiantes durante la crisis sanitaria de 2020. Recuperado de <https://docs.google.com/spreadsheets/d/e/2PACX-1v TeukpbM sPY4_FPhA0qox5ifrSpuhBofFMGDPN9WmiPoJZALLYwebnVh1w2ENO 0D7Q_wDwCg3Sq8UXN/pubhtml?gid=464625902\&single=true>.

MolinA, L.E. y MesA, F.Y. (2019). Las TIC en escuelas rurales: Realidades y proyección para la integración. Praxis \& Saber, 9(21), 75-98.

<https://doi.org/10.19053/22160159.v9.n21.2018.8924> 
Moral, M.E.; Villalustre, L. y Neira, M.R. (2014). Oportunidades de las TIC para la innovación educativa en las escuelas rurales de Asturias. Aula Abierta, 42, 61-67. <https://doi.org/10.1016/S0210-2773(14)70010-1>

Morales, N. (2018). ICT and pupils of rural areas: Between the digital gap and inclusive education. Bordón: Revista de Pedagogía, 69(3), 41-56. <https://doi.org/10.13042/Bordon.2017.52401>

Mwapwele, S.D.; Marais, M.; Dlamini, S. y Van Biljon, J. (2019). Teachers' ICT Adoption in South African Rural Schools: A Study of Technology Readiness and Implications for the South Africa Connect Broadband Policy. The African Journal of Information and Communication, 24, 1-21. <https://doi.org/10.23962/10539/28658>

Ortega, J.; Lorenzo, M. y Fuentes, J. (2005). Tecnofobia como déficit formativo: Investigando la integración curricular de las TIC en centros públicos de ámbito rural y urbano. Educar, 36, 169-180. $<$ https://doi.org/10.5565/rev/educar.203>

Pescador, C.M. y Valentini, C.B. (2019). Inclusão digital em uma escola do campo: Movimentos provocados a partir da implantação de uma política pública no modelo 1:1. Revista Iberoamericana de Educación, 79(1), 135-154. <https://doi.org/10.35362/rie7913409>

Petko, D.; Cantieni, A. y Prasse, D. (2017). Perceived Quality of Educational Technology Matters. Journal of Educational Computing Research, 54(8), 10701091. <https://doi.org/10.1177/0735633116649373>

Raso, F.; Hinojo, M. y Sola, J. (2015). Integración y uso docente de las tecnologías de la información y la comunicación (TIC) en la escuela rural de la provincia de Granada: Estudio descriptivo. REICE: Revista Electrónica Iberoamericana sobre Calidad, Eficacia y Cambio en Educación, 13(1), 139-159.

Rus, M.; Sandu, M. y Tasen e, T. (2020). Covid-19 crisis in Romania: Between perception and attitude. Technium Social Sciences Journal, 6(4), 69-87.

Skryabin, M.; Zhang, J.; Liu, L. y Zhang, D. (2015). How the ICT development level and usage influence student achievement in reading, mathematics, and science. Computers \& Education, 85, 49-58. $<$ https://doi.org/10.1016/j.compedu.2015.02.004>

Sullivan, K.; McConney, A. y Perry, L.B. (2018). A Comparison of Rural Educational Disadvantage in Australia, Canada, and New Zealand Using OECD's PISA. SAGE Open. Octubre 2018, 1-12. <https://doi.org/10.1177/2158244018805791>

WAng, J.; TigelaAR, D. y AdmiraAl, W. (2019). Connecting rural schools to quality education: Rural teachers' use of digital educational resources. Computers in Human Behavior, 101(6), 68-76. <https://doi.org/10.1016/j.chb.2019.07.009> 